\title{
Olanzapine reduces mania more effectively than divalproex, but has more adverse effects
}

\author{
Tohen M, Baker R, Altshuler L, Zarate C, Suppes T, Ketter T, Milton D, Risser R, Gilmore J, Breier A, Tollefson G. Olanzapine \\ versus divalproex in the treatment of acute mania. American Journal of Psychiatry 2002 June; 159:1011-17.

\section{QUESTION: What is the safety and efficacy of olanzapine compared to divalproex for people with acute bipolar mania?}

\section{Design}

Multi-centre randomised parallel-group trial. Clinicians, assessors and participants were blind to treatment allocation.

\section{Setting}

48 centres in the United States.

\section{Participants}

248 adults hospitalised with acute bipolar manic or mixed episodes, with or without psychotic features (confirmed with Structured Clinical Interview for DSM-IV and minimum score of 20 on the Young Mania Rating Scale at both screening and randomisation); mean age 41 years (range 18-75); $57 \%$ women. Exclusion criteria were unstable or serious medical illness; DSM-IV drug or alcohol dependence within 30 days; history of intolerance to study drugs, and treatment with lithium, anticonvulsants or antipsychotics within 24 hours of randomisation.

\section{Intervention}

3 weeks of treatment with flexibly dosed olanzapine $(5-20 \mathrm{mg} /$ day $)$ or divalproex $(500-2500 \mathrm{mg} /$ day in divided doses). Initial daily doses were $15 \mathrm{mg}$ /day for olanzapine and $750 \mathrm{mg}$ /day for divalproex.

\section{Main outcome measures}

Manic and depressive symptoms measured using mean baseline to endpoint improvement on the Young Mania Rating Scale and the Hamilton Depression Rating Scale;
Beneficial and adverse effects of 3-week treatment with olanzapine or divalproex for acute mania

\begin{tabular}{lcc} 
& $\begin{array}{c}\text { Olanzapine \% } \\
(\mathbf{n}=\mathbf{1 2 5})\end{array}$ & $\begin{array}{c}\text { Divalproex \% } \\
(\mathbf{n}=\mathbf{1 2 6})\end{array}$ \\
Response to treatment & 54 & 42 \\
\hline Remission of mania symptoms & 47 & 34 \\
\hline Somnolence & 39 & 21 \\
\hline Dry mouth & 34 & 6 \\
\hline Increased appetite & 12 & 2 \\
\hline Nausea & 10 & 3 \\
\hline Tremor & 10 & 1 \\
\hline Speech disorder & 8 & 2 \\
\hline Neck rigidity & 7 & 1 \\
\hline Sleep disorder & 6 & 0 \\
\hline Tongue edema & 5 & 3 \\
\hline
\end{tabular}

Note: All reported differences were statistically significant, $p<0.05$. treatment response $(50 \%$ or greater reduction in the mania score); remission (Young Mania Rating Scale endpoint of 12 or less); adverse effects.

\section{Main results}

The mean improvement in Young Mania Rating Scale score was 13.4 points for olanzapine and 10.4 points for divalproex $(\mathrm{p}<0.03)$. Remission and response to treatment were greater in the olanzapine group (table; both NNT 8; p <0.05). There was no difference between groups in depression scores or completion rates. Somnolence, dry mouth and increased appetite were more common with olanzapine. Diarrhea, vomiting and nausea were more common with divalproex.

\section{Conclusions}

Three-week treatment with olanzapine improved mania symptoms and remission compared to divalproex.

\section{COMMENTARY}

Up to $90 \%$ of people with bipolar disorders receive antipsychotics at some time during their illness. Atypical antipsychotics have potential antimanic properties and a low risk of extrapyramidal adverse effects, making them a good choice for bipolar patients who need antipsychotic medication. Olanzapine has recently been approved by the United States Food and Drug Administration for bipolar mania. Tohen $e t$ $a l$, supported by the manufacturers of olanzapine, found that olanzapine improved mania symptoms compared to divalproex. There may be several reasons for this. Firstly, the dosing strategy was more advantageous for olanzapine. Therapeutic dosing takes longer to achieve for divalproex and may postpone clinical response. Secondly, the efficacy of olanzapine may be related to a more favourable adverse reaction profile. Somnolence, the most commonly reported treatment-related adverse effect of olanzapine, may help control agitation in mania.

The prophylactic efficacy and long term safety of olanzapine is unclear. In this 3 week trial, adverse events occurred more frequently in the olanzapine group. Weight gain and abnormal glucose metabolism should be considered when prescribing long term use. Despite these limitations, clinicians treating manic patients will be informed by this paper. Olanzapine shows promising efficacy against mania and has a favourable safety profile. Further studies of olanzapine and other atypical antipsychotic drugs are needed. Mong-Liang Lu, MD MS Department of Psychiatry Taipei Medical University-Wan Fang Hospital Taipei, Taiwan 